\title{
LAS ESCUELAS DE PRIMERA ENSEÑANZA DE SEVILLA DESDE EL SIGLO XVI AL XIX
}

\author{
The Schools of First Letters of Seville from the 16th to the 19th century \\ Jesús Carlos Méndez Paguillo \\ Fecha de recepción: 02/10/2018 \\ Fecha de aceptación: 15/01/2019
}

RESUMEN: Las escuelas de Primera enseñanza, denominadas actualmente como Centros de Educación de Infantil y Primaria, han sido fundamentales en cuanto a la formación inicial que reciben los alumnos, de ahí que pretendamos reconstruir en estas páginas una breve historia de estos centros de enseñanza en la ciudad de Sevilla desde los primeros años del siglo XVI y hasta el final del siglo XIX, cuando se institucionaliza la enseñanza elemental.

PALABRAS CLAVE: Escuelas de Sevilla, Escuelas de Primera Letras, Escuelas Elementales.

ABSTRACT: Primary schools, now called Centers of Infant and Primary Education, have been fundamental in terms of initial training received by students, hence we intend to reconstruct in these pages a brief history of these schools in the city of Seville from the early years of the sixteenth century and until the end of the nineteenth century, when elementary education was institutionalized.

KEYWORDS: Schools of Seville, Schools of First Letters, Elementary Schools.

\section{Introducción}

Al igual que la misión del Museo Pedagógico de la Facultad de Ciencias de la Educación de Sevilla, de recuperar, conservar, archivar y exponer objetos y materiales educativos del pasado, consideramos muy importante recuperar la huella de aquellas escuelas, tanto privados como municipales, que se dieron en Sevilla, en este caso hasta el año 1900, perdurando algunos de ellos en la actualidad.

Este estudio lo hemos creado a través de las fuentes bibliográficas sobre la Historia de la Educación en Sevilla y algunos documentos del Fondo Antiguo de la Biblioteca de la Universidad de Sevilla, intentando contribuir al patrimonio cultural material e inmaterial de estos centros escolares.

\section{Los Comienzos en la Edad Moderna}

Arrancamos nuestra andadura desde principios del siglo XVI, en los inicios de la Edad Moderna y el reinado de los Reyes Católicos, cuando además de la enseñanza individualizada y elitista, que recibían algunos niños de familias de la alta burguesía y nobleza, bajo la influencia del Humanismo europeo con preceptores particulares, existían pequeñas escuelas 
municipales, parroquiales, de beneficencia y otras regentadas por maestros particulares, donde se impartía una enseñanza elemental.

Normalmente, a lo largo de la Historia de la Educación y sobre todo desde principios del siglo XVI, la instrucción que recibían los alumnos (privilegiados), en las grandes ciudades, pasaba por tres etapas: una primera, elemental o de alfabetización, donde se les enseñaba a leer, escribir y contar; una segunda ${ }^{1}$ donde comenzaba la enseñanza del Latín, imprescindible para acceder a los Estudios Generales o universitarios ${ }^{2}$, siendo ésta la tercera, donde se obtenía una licenciatura en Medicina, Teología, Derecho Canónigo o Civil, entre otras.

Las escuelas elementales en esta época dependían, por un lado de los ayuntamientos, que sufragaban los alquileres de locales y el salario a los maestros, y por otro, de maestros particulares que enseñaban en sus propias viviendas, donde acogían a un número reducido de alumnos $^{3}$. En estos casos había que pagar un precio estipulado al maestro ${ }^{4}$, existiendo el contrato de aprendizaje de un año para los alumnos del barrio y el de servicio y aprendizaje para los que se quedaban a vivir internados en la casa del maestro. Normalmente eran alumnos de pueblos, donde además de enseñanza tenían incluida la comida, la ropa y el calzado, (Sánchez Herrero, 2010, p. 36).

En Sevilla, un ejemplo de escuelas particulares lo encontramos en un documento fechado el 30 de octubre de 1501 donde se traspasa una escuela de moços de leer y escribir, propiedad de Diego de Baeza, escribano, vecino de Sevilla del barrio de Santa Cruz, al maestro Francisco de Osorio de la colación de Santa María de Sevilla para enseñar a moços a leer y escribir, (Sánchez Herrero, 2010, p. 34). En general estas escuelas eran unas salas sin condiciones pedagógicas, con bancos corridos adosados a la pared, con unas tablas pupitres para la escritura en cuartillas con el tintero, una alacena para libros, cátedra y silla de maestro, entre otros, (Delgado, 1993, 192).

\footnotetext{
${ }^{1}$ Sin duda, existe el riesgo de confundir las escuelas primarias con los colegios de segunda enseñanza, también llamados colegios menores o escuelas de gramática. Aquí estudiaban Gramática, Retórica y Dialéctica (Trivium), como ciclo medio básico y Astronomía, Música, Geometría y Aritmética (Quadrivium), como ciclo medio superior, (Delgado, 1993, 195).

Algunos de estos colegios religiosos en Sevilla fueron el San Miguel del año 1401, frente a la puerta del mismo nombre en la catedral hispalense o el San Isidoro del año 1530 en el Arzobispado de esta capital. Otros fueron los colegios jesuitas de la Iglesia de la Anunciación (actual Facultad de BB. AA.), el de San Hermenegildo en 1580, el San Gregorio de los seminaristas ingleses en 1591, el San Patricio de los irlandeses, el seminario de San Luis de 1609, junto a las Becas de la Concepción en la calle Jesús del Gran Poder en 1620 (González Moreno, 1991, p. 240).

2 Igualmente citamos de principios del siglo XVI los dos colegios mayores o universitarios sevillanos, el Santo Tomas de Aquino de Diego de Deza del año 1516, que impartía Teología y Filosofía y el colegio Santa María de Jesús de Fernández de Santaella, origen de la Universidad de Sevilla, que estaba autorizado en dar titulaciones de Teología, Filosofía, Medicina y Derecho Canónigo y Civil, para de esta manera no tener que desplazarse a Salamanca, Alcalá de Henares o Valladolid. (Aguilar Piñal, 2015; Sánchez Herrero, 2010). Junto a estos estaban otros colegios mayores residencias de los universitarios, como el Colegio Mayor San Buenaventura, el colegio San Francisco de Paula, el San Alberto de Sicilia, el San Acasio y el San Laureano. También el San José y Santo Ángel, (Ibídem, 2015).

${ }^{3}$ Conocemos de un bachiller de Gramática, ayudante de un maestro del Colegio de San Miguel, que daba clases particulares a 15 alumnos (Sánchez Herrero, 2010, p. 38-39). También de una alumna que fue internada en la escuela de la colación de San Pedro, hija de Silvestre Vento. Y Ruiz Berrio (2004), que nos dice que en algunas clases iban desde los 40 a los 140 alumnos, dependiendo de la fama del maestro.

${ }^{4}$ Durante la segunda mitad del siglo XVI y primera del XVII un maestro cobraba dos reales al mes por enseñar a leer, cuatro reales si se enseñaba a leer y escribir y seis si se añadía la enseñanza de las cuentas (Ibídem, 2004, p. 16; Delgado, 1993, 187).
} 
También conocemos por Guichot y Parody (1990, p. 311), que en 1545 se realizó una petición por parte de los maestros de la enseñanza primera al asistente Marqués de Cortés sobre el deplorable estado en que se encontraba la Instrucción pública, desde la elemental hasta la superior, sostenida por el Ayuntamiento en los años de mayor auge y prosperidad de la capital de Andalucía. En la instancia se solicitan salarios atrasados para dos maestros, casas para enseñar la doctrina católica y que se tuviera diligencia en ver que hacen los maestros. En otra petición exigen que nadie abra escuelas sin licencia, que se sepa si el maestro es de buena vida y que se señale el precio que han de llevar cada mes por enseñar a leer y escribir.

Por otra parte, las primeras escuelas elementales de la Iglesia se crearon por órdenes religiosas, como los escolapios de San José de Calasanz, que fundaría en Roma la primera escuela pública de primeras letras en 1597, estableciéndose en España desde el año 1682 (Aguilar Piñal, 1988, p. 146), o los jesuitas. Ambos desarrollaban su magisterio con niños pobres de manera gratuita ligando la alfabetización con la catequesis, y tomando relevancia desde el concilio de Trento (1546-1563), en cuanto que se pretendía luchar contra las ideas protestantes de Lutero y Calvino, ordenando a obispos y párrocos a abrir escuelas y obligando a llevar el control religioso y moral de las mismas (Ruiz Berrio, 2004, p. 12). Algunas de estas escuelas se ubicaron cerca de las iglesias, en la colación de San Salvador, de San Juan, de San Pedro, Santa Catalina o de Omnium Sanctórum, etc. de ahí que se las llamara también escuelas parroquiales.

Tras esta enseñanza elemental, que podía durar varios años, el alumno podía iniciar su carrera laboral como aprendiz en cualquier taller artesanal de la ciudad o bien entrar en un colegio de segunda enseñanza ${ }^{5}$ aprendiendo el Latín, como lengua oficial tras el Concilio de Trento, para comenzar el seminario y formar parte del clero o continuar sus estudios hasta alcanzar los Estudios Generales o universitarios.

Los niños de aproximadamente unos siete u ocho años de edad, que podían asistir a la enseñanza primera o elemental, recibían del maestro ${ }^{6}$ un aprendizaje básico: primero la lectura con la ayuda de un ayo y de una cartilla universal, a base de leer las letras en voz alta, repetir y memorizarlas. Esta cartilla de lectura tenía dos partes; la primera con abecedario, vocales y consonantes, composición de las consonantes con las vocales (método silábico) y frases sencillas, junto con la tabla pitagórica o de multiplicar, y una segunda parte con la doctrina cristiana de los Mandamientos e Artículos e otras cosas santa e buenas, según el Sínodo de Jaén de 1542 (Sánchez Herrero 2010, p. 49-51). Estas cartillas, de unas 16 páginas, se editaban en la península desde la época de los Reyes Católicos gracias a la expansión de la técnica de la imprenta y se hacían en su gran mayoría en Valladolid y en Sevilla en la imprenta de Alonso de la Barrera, vendiéndose ${ }^{7}$ en estancos por todo el reino de España y América y utilizándose los beneficios que originaba para el mantenimiento de la catedral de

\footnotetext{
${ }^{5}$ A lo largo del siglo XVII los dominicos abrieron un colegio en el convento de San Jacinto (1623), otro fue el de Santo Tomás de 1598 y que junto con los jesuitas del San Hermenegildo de 1580, el San Isidoro de San Miguel y 14 preceptores privados, impartían segunda enseñanza o Gramática Latina a 620 alumnos en Sevilla (Aguilar Piñal, 2015, p. 170).

${ }^{6}$ La enseñanza de la lectura y escritura la realizaban en este siglo: Cambiadores, bachilleres de Gramática, maestro de mostrar a leer y escribir, escribano de enseñar moços, y clérigos (Sánchez Herrero, 2010, p. 38-40).

${ }^{7}$ Los historiadores Viñao y Resines calculan que se vendieron cerca de 70 millones de ejemplares, a pesar de las falsificaciones, desde del siglo XVI hasta principios del siglo XIX. (Rueda Ramírez, 2010). Guichot y Parody (1990), nos informa sobre un vendedor de libros en Sevilla, Baltasar de Reis, que quiere dar a los niños pobres un $A, B, C$ (Abecedario) o cartilla de lectura hecha por él ya que muchos niños no van a la escuela porque ésta vale 10 maravedíes.
} 
Valladolid, que era quien tenía el monopolio de su distribución (Ruiz Berrio, 2004; Rueda Ramírez, 2010).

Un tiempo después, cuando el alumno ya dominaba la lectura, el maestro le enseñaba a escribir, copiar, hacer caligrafías y dictados, junto con las cuatro operaciones de cálculo. Escribían con pluma de ánade, oca, cisne o cuervo, que ellos debían aprender a cortar (García Garralón, 2007, p. 199). Además, su formación debía completarse con nociones de buena conducta y doctrina religiosa. El horario escolar de las escuelas de Madrid según un informe de 1588 era en invierno de 8 a 12 por la mañana y de 2 a 6 por la tarde y en verano de 7 a 11 por la mañana y de 3 a 7 por la tarde (Ruiz Berrio, 2004, p. 21).

Ya en el siglo XVII destacamos en Sevilla al gremio de la Universidad de Mareantes de San Telmo que pondrán en práctica la función de mecenazgo creando el Real Colegio de Mareantes de San Telmo ${ }^{8}$ en 1681, abriendo sus puertas con una Real Cédula de 17 de junio para recibir y educar a unos 150 muchachos huérfanos y pobres que quisieran dedicarse a la marinería. Para ello los alumnos debían tener entre 8 y 14 años y permanecer 8 años en el seminario donde se les enseñaba en las primeras letras y en las técnicas de la navegación de manera teórica y práctica como paje, grumete, marinero y piloto, (García Garralón, 2007).

\section{EI Siglo de las Luces y la Sociedad Económica Sevillana de Amigos del País (SESAP)}

Entrado en el siglo XVIII la preocupación por la enseñanza en general de la población española tendrá un gran impulso bajo el reinado de Carlos III (1716-1788), y sus ministros Floridablanca, Campomanes, Jovellanos y Cabarrús, que junto con las ideas del movimiento de la Ilustración ${ }^{9}$ y las Sociedades Económicas de Amigos del País (SEAP) ${ }^{10}$, procuraran reformas para subsanar el atraso económico, agrícola, en las artes y oficios mecánicos, la industria, navegación y la cultura que padecía el país (Calderón España, 1993). Como dato significativo citamos que la Universidad hispalense volvió a dar grados públicos de doctor en el año 1739, habiendo transcurrido 58 años en los que no se ejecutó tal función, (Aguilar Piñal, 2015, p. 169).

En esta línea, en 1767 los Examinadores de maestros de Sevilla, agrupados en el gremio Arte de las Primeras Letras, enviaron al asistente del reino en Andalucía, Pablo de Olavide, un detallado informe en defensa de las escuelas públicas y en contra de la enseñanza

\footnotetext{
${ }^{8}$ Este establecimiento sevillano cerró en el año 1847 y dos años después el Ministerio de Instrucción Pública lo vendió

a los duques de Montpensier (Aguilar Piñal, 2015, p. 190).
}

\footnotetext{
${ }^{9}$ El movimiento de la Ilustración se dio en Europa en el siglo XVIII (Siglo de las Luces), y estuvo representado por miembros de la burguesía, de los que destacaron: Locke, Montesquieu, Voltaire, Rousseau... En España coincide con el reinado de Carlos III. Consistía en la búsqueda de la verdad a través de la razón y en someter a crítica aquéllas que se basaban en la tradición, la superstición o la ignorancia. Buscaban el conocimiento enciclopédico, los avances tecnológicos en la agricultura e industria y la mejora de la enseñanza y la cultura para el desarrollo y crecimiento de los países. Entre los ilustrados españoles destacó el grupo sevillano con el adelantado Pablo de Olavide, junto a Melchor Gaspar de Jovellanos.

${ }^{10}$ En general, las SEAP eran agrupaciones de personas de alta posición y distintas profesiones que estaban interesadas en los avances científicos, las reformas en la agricultura, la industria, el comercio, la navegación, las novedades literarias y la educación, y que sirvieron al gobierno de Carlos III para proyectar las "luces" y fomentar el desarrollo económico. La primera SEAP fue la de las Vascongadas, creada por Floridablanca en 1764 y se expandieron a raíz del libro de Pedro R. de Campomanes en 1774, Discurso sobre el Fomento de la industria popular.
} 
privada no controlada, hablando de los maestros ayos (intrusos) que no han superado el riguroso examen para obtener el título. También exigen control anual de los examinadores de las escuelas y sus maestros, una paga decorosa y estable, y la creación de una academia donde puedan reunirse los 200 maestros existentes en la capital, con los ayos y leccionistas, (Ibídem, p. 171-172).

Por su parte, La Sociedad Económica Sevillana de Amigos del País (SESAP), se denominada en sus comienzos Sociedad Patriótica, y aprobó sus estatutos en $1777^{11}$. Solían reunirse en la residencia del asistente Pablo de Olavide, en los Reales Alcázares, para hablar de las novedades literarias del extranjero y ver el modo de favorecer el fomento de la industria, la agricultura, las artes y el comercio, a través de la enseñanza a la juventud de las matemáticas, con escuelas de cultura y primeras letras, con nuevas técnicas de hilaturas, laboratorio de química y escuela de instrucción para parteras (Aguilar Piñal, 2015; Calderón España, 1993, p. 53).

Estas Sociedades también realizaron informes sobre distintos aspectos de la sociedad española y sus deficiencias en cuanto a educación ${ }^{12}$. Así tenemos constancia, por los documentos históricos de la Universidad de Sevilla (Fondo Antiguo), de cómo desde la Sociedad Patriótica se demandan más escuelas en Sevilla, sobre todo de niñas, denominadas escuelas de costura (Calderón España, 1993, p. 127).

A este respecto, los primeros centros que conocemos de enseñanza para alumnas fueron el Colegio del Espíritu Santo en el convento de las Agustinas, fundado por el arzobispo Manuel Arias en 1715, para 12 niñas de la nobleza de entre 7 y 16 años, junto al Seminario de niñas de la Santísima Trinidad fundado en 1720 por la madre Isabel Josefa de Santa Rita, siendo de pago para las internas y gratuitas para las niñas del barrio. Aquí se les enseñaba costura, bordado, botonería, medias, calzado de mujer y a escribir, contar, dibujo y música (Yanes Cabrera, 2002, p. 83).

Junto a éstas, la Sociedad Patriótica en 1778, como decíamos antes, ya había intentado abrir escuelas de hilado de lino, lana y algodón, con enseñanzas de doctrina cristiana, buenos modales y nociones de lectura y escritura, en unos locales de la calle Becas de la Alameda, otra entre Puerta Osario y Carmona y por último, en Triana. Aunque al final solo se consiguieron dos; en el barrio de San Lorenzo y en el de Triana, con 12 alumnas cada una (Calderón España, 1993, p.108).

Y por petición de una Real Cédula de S. M. expedida en Aranjuez el 11 de mayo de 1783 , se comunica que han puesto en práctica una escuela más de niñas de coser en el barrio de San Gil. En el documento de Instrucciones de esta escuela (Fondo Antiguo de la U.S. y Calderón España, 1993: 131), se detalla que se debe enseñar a las niñas principios de la religión católica y recta moral, para que puedan constituirse en dignas madres de familia, útiles a los suyos y al Estado, además de labores de mano como costura, hacer calcetas,

\footnotetext{
${ }^{11}$ Estatutos de la Real Sociedad Patriótica de la Muy Noble y Muy Leal ciudad de Sevilla y su reynado, con fecha del 16 de diciembre de 1777. Fondo antiguo Digitalizado de la Universidad de Sevilla.

${ }^{12}$ Las profesoras Corts y Calderón (2008), también nos hablan de un discurso leído por Isidoro Morales Rodríguez en la Junta General de la Sociedad Patriótica de Sevilla en el año 1789 y de una Memoria sobre educación elaborada por Agustín María de la Cuadra González y presentada a la Sociedad Económica Sevillana de Amigos del País (SESAP), en el año 1846 donde diserta sobre los beneficios de la educación pública afirmando que: "Los pueblos pueden ser sabios antes que ricos, con tal que sean libres".
} 
medias, redecitas y enseñar a leer a aquellas niñas que muestren inclinación para ello, con sueldo para la maestra y capacidad para 30 alumnas pobres entre los 6 y 7 años de edad.

En un segundo documento del año 1785, la Sociedad Patriótica dice que esta escuela de costura de San Gil ha funcionado tan bien que piden exhortación para crear más escuelas gratuitas de niñas en Sevilla y arrabales, en concreto: En Triana en la parroquia de Santa Ana y la O; en la Iglesia Mayor; en la puerta del Arenal, Carretería y Baratillo; en la Magdalena en el barrio de la Cestería; en San Vicente en los Humeros; otras cerca de Omnium Sanctórum, San Roque y San Bernardo. Continúa pidiendo otras escuelas de niñas que pudieran acoger a razón de 15 alumnas por clase con una gratificación para las maestras de 50 ducados, que era lo que se pagaba en la Corte (Calderón España, 1993, 134), y que se ubicaran en las parroquias de San Julián, Santa Marina, San Marcos, Santa Lucía, San Román, Santa Catalina, Santiago, San Esteban, San Ildefonso, Bartolomé, San Nicolás, San Isidoro, Santa María la Blanca, Santa Cruz, San Pedro, San Juan de la Palma, San Martín, San Andrés, El Salvador, San Lorenzo y San Miguel.

En el año 1790 estas escuelas de costura de la Sociedad Económica Sevillana de Amigos del País, pasaron a denominarse escuelas "amigas ${ }^{13}$ ", estando regentadas por personas dedicadas a la enseñanza elemental desprovistas del correspondiente título (Calderón España, 1993, p. 128). Estableciendo para el año 1791 un número de 24 maestras amigas en la ciudad, aunque esta cifra continuó aumentando con los años y no todas estuvieron subvencionadas. Además de las escuelas de niñas, la SESAP también ayudó a las escuelas de niños con el alquiler de viviendas, mobiliario y salarios a los maestros de San Andrés, Triana, San Bernardo, San Roque (Ibídem, 1993, p. 156).

Junto a la preocupación de estas Sociedades por la educación de los más pequeños, también había particulares que durante este siglo XVIII donaron importantes cantidades de dinero para la construcción de escuelas gratuitas para niños pobres y que luego se las ofrecieron a algunas órdenes religiosas para su dirección. Como por ejemplo el colegio San Luis de la Salle, en la calle del mismo nombre, que se construyó en el año 1765 en unos solares alrededor del noviciado de San Luis, (la iglesia finalizaría su construcción en 1732). Esta obra caritativa fue de don Nicolás de Robles, comerciante de paños, continuándola después su viuda doña Dionisia Encinas: "compadecidos por la indigencia material y espiritual en la que vivían los niños de tan numerosa como pobre barriada" (Aguilar Piñal, 1988, p. 167). Sobre la situación de los niños y niñas de Sevilla podemos citar el comentario del padre Calatayud, jesuita que predicó en Sevilla y su entorno y en una de sus predicaciones insistió en: "...la rudeza e ignorancia en la doctrina cristiana que había experimentado en seis barrios o parroquias de Sevilla, como: Santa Marina, Omnium Sanctórum, San Gil, San Román, San Marcos y Santa Lucía”, (Ruiz Carnal, 2001, p. 37).

Hay que recordar que, un par de años después, en 1767 los jesuitas ${ }^{14}$ fueron expulsados de España y América, siendo vendidos algunos de sus edificios, otros pasaron a

\footnotetext{
${ }^{13}$ Este formato de escuelas llegó a sobrevivir hasta principios del siglo XX. Sánchez Pizjuán, en un informe de 1899 nos cuenta que:" Hay otro tipo de casas que recogen a niñas pobres durante algunas horas del día que les llaman amigas y cuyas dueñas, mujeres pobrísimas las llaman pomposamente academias. Las describe como casas húmedas, sucias y pestilentes, rogando que desaparezcan las 32 que hay en Sevilla (Langa, 2001, p. 82).

${ }^{14}$ A los jesuitas se les expulsó de España y de América en el año 1767 ya que se les acusó de haber instigado y participado en la conocida revuelta contra el marqués de Esquilache, ministro de hacienda de Carlos III, así como de ser un obstáculo para la expansión de las ideas de la Ilustración. En el año 1798, el rey Carlos IV terminó de expropiar los bienes de los jesuitas.
} 
otras ordenes religiosas o se convirtieron en hospicios, como el edificio de Indias, donde se trasladó el Seminario de los niños Toribios, para instruir en él a niños en primeras letras, doctrina cristiana, oficios mecánicos y como "corrección" para jóvenes inquietos (Aguilar Piñal, 2015, p. 70 y 194). Esta institución fue tribunal tutelar y una adelantada de su tiempo, ya que hasta 1887 no surgiría otra similar en Estados Unidos, denominada George Junior Republic, (Corts y Calderón, 2008: 19).

Fray Gabriel Baca escribió en 1766 el libro Los Toribios de Sevilla en el que se relata la creación de un reformatorio por parte de un terciario franciscano procedente de Asturias, Toribo de Velasco, en la Plaza del Pumarejo de Sevilla acogiendo a niños abandonados, ladronzuelos, vagos y desprotegidos (Montero Pedrera, 1993; Corts y Calderón, 2008).

Con respecto a la formación de los maestros, el primer gremio dedicado a estas enseñanzas fue la Hermandad de San Casiano de Madrid, fundada en 1642, obteniendo la consideración del magisterio de las escuelas de primeras letras. En una de sus acciones limitó el número de escuelas, a fin de que la multiplicidad hiciera poco rentable el ejercicio de la profesión. En 1727 el gremio de Sevilla del Arte de las Primeras Letras, solicitó la misma consideración quedando reducidas las escuelas de la ciudad a 31 (Aguilar Piñal, 1988, p. 162). La Hermandad de San Casiano pasó a denominarse Colegio Académico de Primeras Letras desde 1780 hasta el año 1791, cuando se suprime debido a los vicios en privilegios y favoritismo a parientes, constatándose que en Sevilla solo había 33 maestros de escuela, por lo que se dio libertad de oficio (Aguilar Piñal, 2015, p. 63). A partir de entonces se crea la Academia de Primera Educación para la preparación y autorización de maestros dependiente de la Sociedad Económica de Amigos del País y un Reglamento de Escuelas Primarias, que puede considerarse como el primero que se conoce en la historia escolar española y trataba sobre la administración e inspección de escuelas, el régimen interno de las mismas y todo lo concerniente a los maestros. Dejando en 25 el número de escuelas de la SESAP, junto con otras escuelas públicas municipales y las religiosas.

También se fijaba un horario lectivo de verano desde las 7 a las 11 por la mañana y de 4 a 6 por la tarde y en invierno de 8 a 11 por la mañana y de 2 a 4 por la tarde. La edad de admisión a la escuela se fija en 5 años, así como los libros a utilizar y las fechas de exámenes.

\section{La institucionalización de la Enseñanza primaria}

El siglo XIX marca el tránsito de la Edad Moderna a la Contemporánea, traducido en el triunfo político que supuso las ideas del liberalismo frente a las absolutistas, con la división de poderes y destacando la burguesía como clase social dominante. Referente a la educación, será cuando la enseñanza pasará a estar regulada por el Estado, retirando estas obligaciones o competencias a la Iglesia y a las SEAP y reflejando en la Constitución de Cádiz de 1812 los principios básicos del liberalismo sobre la Instrucción: escuela pública de primeras letras en todos los municipios de España, gratuita, uniforme y libre. En el texto constitucional ya se establece la idea de educación como un conjunto de propósitos donde el Estado debe intervenir como un bien común, tanto en su organización, financiación y control, siendo la base del sistema educativo español con el Plan de Estudios de 1813, conocido como Plan Quintana ${ }^{15}$.

\footnotetext{
${ }^{15}$ Este Plan tuvo que tener como base "La Memoria sobre la Educación Pública en las escuelas y colegios de niños" de 1802 y el "Plan General de Instrucción pública" de 1809 del ilustrado M. G. de Jovellanos.
} 
Sin embargo, los municipios apenas destinaron presupuestos para nuevas escuelas y el balance escolar con el que comienza el nuevo siglo era el siguiente: en 1815 en Sevilla solo existían 40 escuelas $^{16}$, a las que acudían unos 2.000 alumnos, cuando eran más de 4.000 los niños que estaban en edad de frecuentarlas, según José Vargas Ponce en su discurso pronunciado en la SESAP y titulado "El estado de las escuelas de Primeras letras en Sevilla". La suma sale de contar los alumnos de las escuelas de conventos regulares como el de la Cartuja, con 60 niños; el de Santo Tomás con 223; el de San Jacinto con 20; San Agustín con 115; El Pópulo con 150 y los Trinitarios Descalzos con 30, más otros 1.300 niños que acudían a escuelas gratuitas financiadas por la SESAP, a escuelas parroquiales y escuelas de maestros particulares", (Ruiz Carnal, 2001, p. 113-114).

El regreso de Fernando VII al trono supondrá la vuelta al absolutismo y para ello derogará el Plan Quintana por un nuevo Reglamento de Primeras letras, e introduciendo el método de enseñanza de lectura y escritura conocido como Bell-Lancasteriano o Enseñanza Mutua $^{17}$, llegando incluso el monarca a organizar una Escuela Central de maestros para difundir esta metodología en 1819, (Real Apolo, 2012, p. 74).

Durante el Trienio Liberal, de 1820 a 1823, se recuperó el Reglamento General de Instrucción Pública de Quintana, aunque con algunas reformas, y se establecieron oficialmente las tres etapas escolares; Primera, Segunda y Tercera. También se diferenciaba la enseñanza pública (la sostenida por ayuntamientos, benefactores, fundaciones o pías), de la privada religiosa. Pero, de nuevo, tras el triunfo del absolutismo con la llegada de los Cien mil hijos de San Luis, Fernando VII retomará el gobierno durante la 'década ominosa' implantando el Plan de Estudios y Reglamento de la Escuelas de Primeras Letras del Reino, conocido como Plan Calomarde de 1825, que se rige por la uniformidad en los libros, materias, métodos, premios y castigos, exámenes y prohibiendo la coeducación. La enseñanza de las niñas se limitaría a las labores domésticas y Doctrina Cristiana (Real Apolo, 2012, p. 75-76).

Con Isabel II (1833-1868), las políticas educativas oscilan según quién esté en el gobierno: moderados o liberales. Los primeros presentan el Plan de estudios del Duque de Rivas que sirvió para redactar la Ley de Instrucción Primaria del año 1838, del marqués de Someruelos. La novedad es que introduce por primera vez la educación de párvulos ${ }^{18}$ y la de

\footnotetext{
${ }^{16}$ Hay una gran dificultad en realizar un recuento de escuelas en Sevilla propiamente dicho, ya que los informes y datos oficiales aplican el término escuela a un aula con capacidad para 50 alumnos. Por ello la mayoría de escuelas eran unitarias, recibiendo el nombre del barrio, colación o del maestro. Habrá que esperar hasta principios del siglo XX cuando se crean las Escuelas Graduadas, es decir, escuelas con tres secciones o clases y los Grupos Escolares, con 6 aulas o más, llegando a tener algunas hasta 22 aulas, siendo ya reconocidos los colegios por un número, nombre o título.

${ }^{17}$ Joseph Lancaster publicó en 1803 el libro Mejoras en la Educación, en las que proponía un nuevo sistema de enseñanza a bajo coste. Tuvo éxito en Inglaterra y en los países latinoamericanos. Consistía en colocar a los niños sentados en filas en un gran salón y en un extremo de estas filas se situaba un niño monitor y al frente de todos el maestro que impartía la lección. Después los monitores de cada fila repetían la clase a los niños. El método de Enseñanza Mutua lo llevó a cabo el reverendo Bell, también en Inglaterra, pero en 1797, cuando recibió el encargo de enseñar a niños de soldados. Un día vio como uno de ellos enseñaba a otros escribiendo en la arena y se propuso enseñar solo a los más diestros para que estos enseñaran al resto con cajas o bandejas de arena, (Sanabria, 2010).
}

${ }^{18}$ La educación de párvulos se circunscribía a unas casas con el mismo nombre que las escuelas de niñas de la SESAP: "amigas". Era la vivienda de una vecina, donde las mujeres que trabajaban podían dejar a sus hijos en modo de guardería, según nos cuenta el maestro de párvulos sevillano Faustino Álvarez Sáenz, (Loscertales y Navarro, 1995, p. 77). Sobre la denominación de escuelas de párvulos también encontramos otra definición en las obras del padre Manjón, pero como escuelas “migas” en su libro El Pensamiento del Ave-María. Tercera 
adultos (capítulo IX). También divide la enseñanza Primaria en Elemental (Lectura, Escritura, Elementos de Gramática Castellana, Ortografía, Aritmética y principios de Religión y Moral), y en Primaria Superior (Historia y Geografía de España, Aritmética Avanzada, Física e Historia Natural y Dibujo Lineal). Obligando a los pueblos de más de 100 habitantes a sostener una de ellas y a las villas de más de 1.200 vecinos a mantener una de Primaria Superior, éstas solo gratuitas para los niños pobres. También establecerá la Escuela Normal ${ }^{19}$ Central de Madrid en 1838 y el Reglamento de Instrucción Primera impulsada por Pablo Montesinos $^{20}$. En Sevilla la Escuela Normal se fundó en el año 1845 en la calle Don Remondo y en 1859 lo hizo la Escuela Normal de Maestras ${ }^{21}$, en la calle Jesús del Gran Poder (Pérez González, 1982, p. 16), con lo que el Estado se erigía en formador de docentes introduciendo la pedagogía de Comenius, Pestalozzi, Rousseau, Fröebel, etc.

En el año 1844 se aprueba el Plan Pidal que regulaba las enseñanzas medias y superiores (Real Apolo, 2012, p. 78). Y en 1857 la Ley de Instrucción Pública, popularmente conocida como Ley Moyano, consolidando el sistema educativo español en todo su conjunto, manteniéndose vigente durante 113 años, concretamente hasta la Ley Villar Palasí de 1970. Fue una Ley de rápida y fácil aprobación en las Cortes, pues estaba elaborada con los principios sociopolíticos de moderados y liberales (Real Apolo, 2012, p. 80), limitándose a ordenar lo que ya se había decretado y no a reformar (Ávila Fernández, 1989-1990, p. 226). La Ley reguló el curriculum de la enseñanza Primaria o Elemental obligatoria, pero gratuita solo para los que no pudieran pagarla, y abarcará a los alumnos con edades de 6 a 10 años. La Ley establecía la unión de la enseñanza Elemental con la Primaria Superior, ampliando en un año la edad obligatoria de ingreso en las escuelas: de 5 a 10 años en los lugares donde no hubiera escuelas de párvulos. Destacó también por la introducción del Canto como materia (Ibídem, 1989-1990).

Sin embargo, donde hubo grandes diferencias entre los partidos políticos fue con la enseñanza superior o universitaria, siendo uno más de los factores que provocarían el rechazo a la monarquía de Isabel II en 1868 y la llegada de la I República española.

parte. Modos de enseñar, 1935, p. 56. Aquí nos la describe como: "Llámese a la pobre mujer que recoge las migas o migafillas de la humanidad, lo más ruin e inservible (niños y niñas de 3 y 4 años), y por unas migas (popularmente un chavico o un centimillo), los cuida y limpia, les da agua y les enseña la letra A y el Padrenuestro". También Luis Bello en su libro Viaje por las escuelas de Andalucía, cita las escuelas "migas" como pequeñas escuelas que algunos maestros tenían en sus casas (1998, reedición de 1929, p. 292).

\footnotetext{
${ }^{19}$ Se llamaban escuelas Normales porque su funcionamiento debía de ser norma para todas las demás. Su influencia viene de Prusia y Francia. Éstas daban el título de maestro y maestra tras un examen de ingreso y cuatro cursos, siendo el tercero y cuarto con prácticas en la Escuela aneja o en otras si hubiera exceso de alumnado, con un examen final.
}

${ }^{20}$ En el Reglamento de Escuelas Públicas de Instrucción Primera Elemental de 26 de noviembre de 1838, conocido como Reglamento Montesinos, recomienda que la enseñanza de la lectura y escritura sea simultánea y de manera comprensiva, criticando la enseñanza mecánica de la lectura. En la escritura debe aspirarse a que consigan una letra igual, clara sin especiales adornos. Recomienda material de enseñanza de uso colectivo como libros, lápices, papel, pizarras, tabletas, así como bancos de arena. El maestro será quien elija los libros de texto previa autorización de la Junta Local. Considera importante la formación religiosa y moral y el aseo y la higiene, el no prodigar los premios y ser moderados con los castigos, (Ávila Fernández, 1989-1990, p. 223).

21 “Pascual Madoz recoge en su Diccionario Geográfico Estadístico Histórico (1845-1950), que Sevilla tenía menos escuelas de niñas que de niños, pero que sin embargo había un alto número de Colegios de Señoritas (hasta 67), en los que se imparte: Religión, Moral, Urbanidad y se les inculcan ideas fijas de piedad cristiana, decoro, buen tono, lectura, caligrafía, gramática castellana, aritmética, Geografía e Historia, idiomas, costura y bordado", (Flecha, 2002, p. 260). 
Con la Restauración de la monarquía de Alfonso XII, se ponía fin al Sexenio Revolucionario, alternándose indistintamente en el gobierno conservadores y liberales, hasta el año 1923, manteniendo un periodo de relativa normalidad, ya que los conservadores intentarán retomar la línea conflictiva con respecto al estamento universitario de antes del Sexenio, imponiendo la obediencia a la monarquía y anulando la libertad de cátedra, hecho que provocó la dimisión de numerosos catedráticos y profesores universitarios. Uno de ellos, Francisco Giner de los Ríos, fue el promotor de una enseñanza alternativa e innovadora, frente a la enseñanza tradicional, llevándola a la práctica desde la Institución Libre de Enseñanza (ILE), en consonancia con lo que se realizaba en el ámbito educativo europeo y difundiendo las ideas del krausismo, que pretendían a través de la educación transformar a la sociedad. Además, fueron muy combativos en los debates de los Congresos Pedagógicos, como el de 1882, criticando la insalubridad de las escuelas y demandando inspecciones sanitarias (Añón, 2005, p. 38). También estaban en contra de escuela de párvulos tradicional española defendida por Pablo Montesinos frente la moderna fröebeliana de los jardines de Infancia defendida por los partidarios de la ILE, (Loscertales, 1995; Palacios Bañuelos, 1988).

De este siglo XIX fueron también los colegios de San Diego de 1841 donde impartió clases Alberto Lista ${ }^{22}$, el colegio de la calle Mesón del Moro de 1845 (actual CEIP San Isidoro), y el que había en el convento de San Jacinto, adquirido por el Ayuntamiento tras la desamortización de Mendizábal, considerados los primeros colegios en edificios de propiedad municipal de Sevilla (AA. VV., 2008). De este año también fue el Instituto Provincial y Universitario de Sevilla, primer instituto oficial de enseñanza secundaria en la ciudad, trasladándose en el año 1867 al convento de San Pedro de Alcántara en la calle Amor de Dios (Yanes Cabrera, 2002). También abrieron los colegios de párvulos Santa Justa y Rufina de 1849 y del Omnium Sanctórum de 1853, (Cuenca Toribio, 1991, p. 174).

Nicolás Salas (1991, p. 111), cita además a otros colegios religiosos en estos años como el San Joaquín (Carmelitas de la Caridad), el San José, las Hermanas de la Cruz, el Salvador, las Hijas de María, el Hospital de San Lázaro, el Santa Isabel en la calle Pagés del Corro, el instituto María Reparadora de la Asociación del Buen Pastor...

También hay constancia de que los jesuitas regentaron un colegio en la calle Argote de Molina desde 1871 hasta el 1882, pero acabó trasladándose a Málaga.

Por otra parte, y similar al mecenazgo del colegio de San Luis, la marquesa de Villanueva compró el convento del Valle para construir el Colegio de Religiosas Sagrado Corazón conocido popularmente como colegio El Valle en el año 1866. Unos años después, en 1887, Dolores Armero Benjumea (protectora también de las Escuelas gratuitas de San Pedro de Triana), compró y cedió el antiguo templo de San Francisco de Paula Tarín, en calle Jesús del Gran Poder, a los jesuitas para crear el Colegio de coristas.

Por su parte, la Diputación de Sevilla creó el colegio de Sordomudos y Ciegos en $1873^{23}$ a instancia de Manuel Pichardo y Casado, su primer director (Salas, 1991 (1), p. 131),

\footnotetext{
${ }^{22}$ Alberto Lista (Sevilla 1775-1848). Con 13 años ocupó una cátedra de Matemáticas de la SESAP, un puesto análogo en la escuela de San Telmo y también fue profesor de Filosofía en el Colegio de San Miguel. En 1804 se ordena sacerdote y fundó el periódico El Espectador Sevillano, exiliándose durante cuatro años por ser considerado como afrancesado. Tras su regreso se instala en Pamplona, Madrid, Sevilla y Cádiz dedicándose a la enseñanza. Fue nombrado canónigo de la Catedral de Sevilla dos años antes de fallecer (Corts y Calderón, 2008, p. 119).

${ }^{23}$ En 1803, la SESAP abrió un establecimiento de sordo-mudos a instancias del párroco de escuelas pías José Navarrete, y el maestro de primeras letras Juan de la Vega Campuzano.
} 
quien leería una Memoria relativa a su Historia, Organización, Régimen y Estado actual en la Exposición Universal de París de 1889, (Corts y Calderón, 2008, pp. 155-159).

Unos años después, en 1886 don Eugenio Fernández de Zendrera, párroco de San Lorenzo, profesor de Religión en la Escuela Normal de Maestros de Sevilla, miembro de la Junta Local de Primera Enseñanza del Ayuntamiento ${ }^{24}$ y visitador de escuelas públicas presentó un informe sobre la Instrucción Primaria municipal donde subraya que en la ciudad de Sevilla, con 132.789 habitantes, debería haber 67 escuelas de niños y el mismo número de niñas, entre públicas y privadas según la Ley Moyano, por lo que reclama que el consistorio subsane la falta de 20 escuelas de niños y 32 de niñas. Advierte sobre el hecho de que las pocas escuelas que existen están muy distantes unas de otras, existiendo barrios como el centro de la ciudad, sin ninguna escuela municipal. También indica que la ciudad tiene 3.389 niños y 3.435 niñas en edad escolar y tan solo asisten a las clases 1.131 niños y 954 niñas, (Corts y Calderón, 2008, p. 97-102).

Destacamos también el colegio privado y laico San Francisco de Paula de 1887, que en la actualidad continúa impartiendo enseñanzas, igual que el colegio religioso Calasancio Hispalense (Padres Escolapios o Escuelas Pías), de 1888 y ubicado en la Plaza Ponce de León hasta el año 1974 cuando se traslada al barrio de Montequinto ( $A B C$ Sevilla 28/08/1974, p. 35-36).

Otra institución sevillana de carácter filantrópico fue la Real Maestranza de Caballería, que financió la construcción del colegio Macarena o Altos Colegios ${ }^{25}$ (calle Feria con Resolana), en el año 1892, por lo elevado de su cimentación y que dos años después cedió al Ayuntamiento. Otros benefactores con carácter particular que donaron a la ciudad edificios y rentas para este fin fueron doña Carmen Benítez y Cortina, que construyó el edificio escolar que lleva su nombre desde 1896. También, en la obra de Luis Bello, Viaje por las escuelas de Andalucía, se cita la iniciativa de don Ramón Galíndez, alcalde republicano que donó un gran legado para construir escuelas laicas, aunque su testamento sería impugnado.

Por último, en 1897 el ayuntamiento sevillano inició las obras para convertir un edificio de las Hermanas de la Caridad en el colegio San Bernardo, terminando sus obras en 1908 (Añón, 2005, p. 89).

Y cerramos este estudio con los datos escolares de Sevilla que obtenemos de las fuentes bibliográficas a falta de censos oficiales y teniendo en cuenta que se contaba como escuela la unidad escolar de una clase con ratio de 50 alumnos. El primer dato pertenece al año 1723 cuando existían 31 escuelas según el gremio del Arte de las Primeras Letras. En 1815 las escuelas religiosas de conventos más las de la Sociedad Económica Sevillana Amigos del País, las parroquiales y de particulares, sumaban 40. En el año 1886 había 47

\footnotetext{
${ }^{24}$ La profesora Montero Pedrera nos detalla todos los organismos encargados de las escuelas primarias en su obra de 1996, La enseñanza Primaria Pública en Sevilla: (1857-1900). En ella nos describe a la Junta Local de Instrucción Primaria, institución creada a mediados del s. XIX y formada por el alcalde, el párroco designado por el obispo y dos o tres miembros de familias nombrados por el gobernador, cuya función era encargarse de todo lo relativo a las escuelas; locales, materiales, nombramiento de maestros, etc. Después pasó a denominarse Junta Local de Primera enseñanza.

${ }^{25}$ El colegio fue inaugurado por la reina María Cristina y su hijo Alfonso XIII en 1892, con motivo de la efemérides del 400 aniversario del descubrimiento de América. La obra pertenece al arquitecto Francisco. Aurelio Álvarez y destaca en una de sus aulas frescos del pintor Antonio Cavallini. El primer director de este centro fue Faustino Álvarez y Sáenz (1894-1910), quien escribió “Apuntes pedagógicos referentes a la Educación de Párvulos en España”.
} 
escuelas de niños y 35 de niñas, faltando 52 escuelas para completar las plazas escolares existentes. En 1896 Sevilla disponía de 12 escuelas municipales masculinas, 10 femeninas, 7 de párvulos e idéntico número de adultos, en tanto que las privadas alcanzaban 18 en la enseñanza de niños y 74 en la de niñas (Cuenca Toribio, 1991, p. 226). Sánchez Pizjuán nos informa que en 1899 en Sevilla existían 171 escuelas de las cuales solo 42 eran municipales (Langa, 2001, p. 81).

Esta era la situación con la que la enseñanza primaria pública arrancará en el siglo XIX en Sevilla, con una población desilusionada por la pérdida de las últimas colonias españolas: Puerto Rico, Cuba y Filipinas, y manipulada por el caciquismo y los pucherazos electorales del sistema político de la Restauración. Hechos que recogieron en sus textos los autores de la Generación literaria del 98 y el movimiento intelectual del Regeneracionismo, convencidos de poder arreglar el atraso que padecía el país, con: "escuela y despensa", según palabras del político Joaquín Costa, que definían perfectamente los problemas más acuciantes en la población española, donde además de la pobreza y analfabetismo (el 48,07\% de una población de 148.315 personas eran analfabetas en Sevilla en el año 1900), (Langa, 2001, p. 81), la educación elemental seguía estancada, con locales arrendados para escuelas mal acondicionados y sin llegar a toda la población escolar por haber pocas escuelas y estar mal repartidas por la ciudad.

\section{Conclusiones}

Un recorrido por la Historia de la Educación Primaria en Sevilla y las escuelas a lo largo de los siglos XVI y XVII nos lleva a reflexionar sobre la poca consideración que esta etapa educativa tuvo frente a la superior o universitaria. Además, mientras los descendientes de la alta burguesía y nobleza gozaban de una educación particular en sus residencias o palacetes, la escuela pública como tal, aparece en primer lugar como un lugar donde recoger a una parte de los niños pobres y ociosos que deambulaban por las calles, más que dedicados a la formación elemental de la lectura, escritura y cálculo. Asumiendo estas competencias la iglesia y en menor medida los municipios.

Afortunadamente, el siglo XVIII marcará una mayor preocupación por esta enseñanza elemental, tanto de las instituciones civiles y gobernantes, siendo el XIX cuando comienzan a construirse las primeras escuelas públicas en edificios municipales, desde escuelas unitarias a graduadas y grupos escolares, abandonando los viejos locales arrendados y en pésimas condiciones para la enseñanza. A ello también contribuyeron los informes y memorias de distintas personalidades y benefactores mostrando su preocupación por el déficit de escuelas elementales con el que combatir las altas tasas de analfabetismo y su interés por elevar la formación y cultura de la población con menos recursos, aunque habrá que esperar hasta el último tercio del siglo XX cuando se alcanza el objetivo de la escolarización total de los niños y niñas.

\section{Referencias bibliográficas}

AA. VV. (2008). Breve Catálogo del Patrimonio Histórico-Educativo de la Escuela Pública Sevillana (Hasta 1950). Sevilla, Ayuntamiento de Sevilla-Educación.

Aguilar Piñal, Fr. (1969). La universidad de Sevilla en el s. XVIII. Estudio sobre la primera reforma universitaria moderna. En Anales de la Universidad Hispalense, 1. Sevilla.

Aguilar Piñal, Fco. (1992). Temas Sevillanos. Primera Serie. Universidad de Sevilla. 
Aguilar Piñal, Fco. (1988). Temas Sevillanos. Segunda Serie. Universidad de Sevilla.

Aguilar Piñal, Fco. (2015). La Sevilla de Olavide. ICAS. Sevilla.

Álvarez Domínguez, P; Núñez Gil, M; Rebollo Espinosa, M. a J. (2012). Viaje hacia una realidad inacabada: El Museo pedagógico de la Facultad de Ciencias de la Educación de la Universidad de Sevilla. Cabás: Revista del Centro de Recursos, Interpretación $y$ Estudios en materia educativa. Consejería de Educación, Cultura y Deporte. Gobierno de Cantabria (España), 8, pp. 157-176.

Añón Abajas, R. M. ${ }^{a}$ (2005). La Arquitectura de las Escuelas Primarias Municipales de Sevilla hasta 1937. Consejería de Obras públicas y Transportes de la Junta de Andalucía y Universidad de Sevilla.

Ávila Fernández, A. (1987). La enseñanza primaria a través de los planes y programas escolares de la legislación española durante el siglo XIX, (Hasta 1820). Revista Cuestiones Pedagógicas, 4, 173-186.

Ávila Fernández, A. (1989-1900). La enseñanza primaria a través de los planes y programas escolares de la legislación española durante el siglo XIX. (Desde el Reglamento General de Instrucción Pública de 1821 hasta la Ley de Instrucción Primaria de 1868). Revista Cuestiones Pedagógicas, 6-7, p. 215-230.

Arbide, J. (2009). Sevilla de maestros y pupitres. Reeditores. Sevilla.

Bello, L. (1998, reedición de 1929). Viaje por las escuelas de Andalucía. Junta de Andalucía, Consejería de Educación y Ciencia, Diputación de Sevilla. Sevilla.

Braojos, Parias y Álvarez (1990). Historia de Sevilla. Sevilla en el Siglo XX. Tomo II. Secretariado de Publicaciones de la Universidad de Sevilla.

Calderón España, M. ${ }^{a}$ C. (1993). La Real Sociedad Económica Sevillana de Amigos del País: Su proyección educativa (1775-1900). Secretariado de Publicaciones de la Universidad de Sevilla.

Corts Giner, M. ${ }^{a}$ I, y Calderón España, M. ${ }^{a}$ C. (2006). Estudios de la Historia de la Educación Andaluza (Siglos XVIII, XIX y XX). Universidad de Sevilla.

Corts Giner, M. ${ }^{a}$ I, y Calderón España, M. ${ }^{a}$ C. (2008). Estudios de la Historia de la Educación Andaluza. Sevilla. Universidad de Sevilla.

Cuenca Toribio, J. M. (1991, reedición de 1976). Historia de Sevilla. Del Antiguo al Nuevo Régimen. Secretariado de Publicaciones de la Universidad de Sevilla.

Flecha García, C. (2002). El saber de las mujeres en Sevilla. En Ciudad y Saber. Sevilla en la Historia de la Educación (pp. 247-278). Kronos, Sevilla.

García Garralón, M. (2007). Taller de Mareantes: El Real Colegio seminario de San Telmo de Sevilla (1681-1847). Vol. 1. Edita Obra Social Cajasol. Sevilla. 
Gómez García, M. ${ }^{a}$ N. (2008). Actualidad del Patrimonio Histórico Educativo Sevillano. La creación del Museo Pedagógico. En Los Cuadernos de la Ciudad Educadora, N. ${ }^{\circ} 1$. El Patrimonio Histórico educativo en el ámbito escolar sevillano.

Gómez García, M. ${ }^{\text {a }}$ N. y Corts Giner, M. ${ }^{a}$ I (2004). Historia de la Educación en Andalucía. Vol. I y II. Sevilla, Centro Cultural El Monte.

González Moreno, J. (1991). Aportación a la Historia de Sevilla. Editorial Castillejo. Sevilla.

Guichot Y Parody, J. (1990, reedición de 1897). Historia del Excmo. Ayuntamiento de Sevilla. Tomo II. Desde Carlos I hasta Felipe V. Edita Colegio Oficial de Aparejadores y Arquitectos de Sevilla.

Hebrard, J. (1989). La Escolarización en los saberes elementales de la época Moderna. Revista de Educación y Ciencia, 288, p. 63-104. Ministerio de Educación. Madrid.

Holgado Barroso, J., Márquez de la Plata, J. M. y Martín Riego, M. (2013). De la Escuela de Magisterio de la Iglesia al CES Cardenal Spínola. Camas (Sevilla), Fondo Editorial de la Fundación San Pablo Andalucía CEU y CEU Ediciones.

Iyanga Pendi, A. (1998). La Educación Contemporánea. Teorías e Instituciones. NAU Llibres, Valencia.

Langa Nuño, C. (2001). Educación y Propaganda en la Sevilla de la Guerra Civil. Una aproximación a través de la prensa. Ayuntamiento de Sevilla. Servicio de Publicaciones.

Loscertales F. y Navarro R. (1995). Faustino Álvarez y Sáenz (1848-1910). Un ilustre maestro de párvulos sevillano. Editorial Alfar, Sevilla.

Manjón A. (1935, reedición de 1902). El Pensamiento del Ave-María. Tercera parte. Modos de enseñar. Granada.

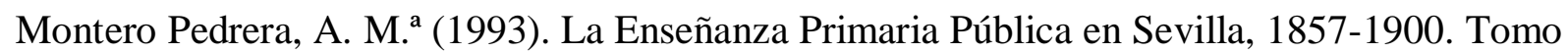
I y II. TD, Facultad de Filosofía y Ciencias de la Educación.

Montero Pedrera, A. M. (1996). La Enseñanza Primaria pública en Sevilla: 1857-1900. GIPES. Sevilla.

Palacios, L. (1988). Instituto-Escuela. Historia de una renovación educativa. MEC. Madrid.

Pérez González, E. (1982). El Magisterio sevillano a comienzos del siglo XX. Ayuntamiento de Sevilla. Servicio de Publicaciones.

Quintana, D. (1975). La Política educativa en España entre 1850-1939. En Historia de la educación en España (1857-1970). Revista de Educación, 240, 30-39. 
Real Apolo, C. (2012). La configuración del Sistema Educativo Español en el siglo XIX. Legislación educativa y pensamiento político. Revista Campo Abierto, 31, (1), 69-94. Universidad de Extremadura.

Rueda Ramírez, P. (2010). Aprender a leer. La enseñanza de las Primeras Letras en el mundo moderno. Revista AH. Andalucía en la Historia, 27, p. 88-91.

Ruiz Berrio, J. (2004). El oficio de maestro en tiempos de Cervantes. Revista de Educación. Ministerio de Educación, Cultura y Deporte. N. ${ }^{\circ} 1$ Extraordinario, p. 11-26. Madrid

Ruiz Carnal, J. (2001). Algunas raíces de las escuelas públicas de Sevilla. Ayuntamiento de Sevilla.

Salas, N. (1991). Sevilla Crónicas XX. Tomo I, 1895-1920. Secretariado de Publicaciones de la U.S.

Sanabria Muñevar, Fco. (2010). Enseñando mutuamente: una aproximación al método Lancasteriano y su proyección en Colombia". Revista de Historia de la Educación de Colombia, 13, (13), 43-76. Universidad de Nariño-Rudecolombia.

Sánchez Herrero, J. (2010). De las Primeras Letras a la Universidad en la Sevilla de finales del siglo XV. Edita S. P. U. S. Sevilla.

Yanes Cabrera, Cristina (2002). Instituto Provincial y Universitario de Sevilla (1845-1868). Génesis del primer centro universitario de educación secundaria oficial. Ayuntamiento de Sevilla. Delegación de Educación 
